\title{
Revealing the mechanisms by which magneto-hydrodynamics disrupts solidification microstructures
}

\author{
B.Cai ${ }^{a}{ }^{*}$, A. Kao ${ }^{\text {b, }}{ }^{*}$, E. Boller ${ }^{c}$, O.V. Magdysyuk ${ }^{d}$, R. C. Atwood ${ }^{d}$, N. T. Vo ${ }^{d}$, K. Pericleous ${ }^{\text {b }}$, P.D. \\ Lee $^{\mathrm{e}}$ \\ a School of Metallurgy and Materials, University of Birmingham, Birmingham, B15 2TT, UK \\ ${ }^{\mathrm{b}}$ Centre for Numerical Modelling and Process Analysis, University of Greenwich, Old Naval \\ College, Park Row, London, SE10 9LS, UK \\ c ESRF-The European Synchrotron, 71 Avenue des Martyrs, 38000 Grenoble, France \\ ${ }^{d}$ Diamond Light Source Ltd, Diamond House, Harwell Science \& Innovation Campus, Didcot, \\ Oxfordshire, OX11 ODE, UK \\ e Mechanical Engineering, University College London, London, WC1E 6BT, UK \\ *Corresponding authors: B.C. b.cai@bham.ac.uk, A.K. A.Kao@greenwich.ac.uk
}

\begin{abstract}
A key technique for controlling solidification microstructures is magneto-hydrodynamics (MHD), resulting from imposing a magnetic field to solidifying metals and alloys. Applications range from bulk stirring to flow control and turbulence damping via the induced Lorentz force. Over the past two decades the Lorentz force caused by the interaction of thermoelectric currents and the magnetic field, a MHD phenomenon known as Thermoelectric Magnetohydrodynamics (TEMHD), was also shown to drive inter-dendritic flow altering microstructural evolution. In this contribution, high-speed synchrotron X-ray tomography and computational simulation are coupled to reveal the evolution, dynamics and mechanisms of solidification within a magnetic field, resolving the complex interplay and competing flow effects arising from Lorentz forces of different origins. The study enabled us to reveal the mechanisms disrupting the traditional columnar dendritic solidification microstructure, ranging from an Archimedes screw-like structure, to one with a highly refined dendritic primary array. We also demonstrate that alloy composition can be tailored to increase or decrease the influence of MHD depending on the Seebeck coefficient and relative density of the primary phase and interdendritic liquid. This work paves the way towards novel computational and experimental methods of exploiting and optimising the application of MHD in solidification processes, together with the calculated design of novel alloys that utilise these forces.
\end{abstract}

Key words: Magneto-hydrodynamics; Solidification microstructure; Alloys; Thermoelectric convection

\section{Introduction}

Magneto-hydrodynamics (MHD) assisted solidification is important for many process techniques such as materials refining [1] or production of alloys and semi-conductors [2-6], and is being explored for new applications such as additive manufacturing [7]. Applied appropriately, microstructural modulation induced by magnetic fields can lead to drastic 
improvements in properties [2-5]. Liquid metal MHD is governed by the Lorentz force $\left(F_{E M}\right)$ and it is well known that applying a static magnetic field changes the melt flow leading to electromagnetic damping [8] or alternatively, a travelling field (including a rotating magnetic field) leads to electromagnetic stirring (EMS) $[9,10]$. A static magnetic field has also been seen to drive flow through its interaction with inherent thermoelectric currents [11-14], generated in solidifying alloys due to spatial variations in temperature and Seebeck coefficient $[11,15-$ 17]. This thermoelectric Lorentz force $\left(F_{T E}\right)$ produces flow in the mushy zone between growing dendrites, an MHD phenomenon known as Thermoelectric Magnetohydrodynamics (TEMHD) $[11,13,15,18] . F_{E M}$ and $F_{T E}$ as defined here, and in the context of crystalline solidification have competing and intertangled ways of driving liquid flow. It is often the case that only one effect is considered at the expense of the other. A better understanding of the relative influence of each of these mechanisms, especially if encapsulated in a computational tool, has the potential to transform our usage of magnetic fields as a parameter in future manufacturing practices.

Many numerical and experimental studies have been carried out to investigate the effect of magnetic fields on solidification. On the experimental side, results show different microstructural effects such as refining grains with increasing magnetic field [3,10,14,19,20], inducing columnar to equiaxed transition [21], and some negative effects, including the appearance of freckles [15], a tilted or irregular solid front [20,22,23], and macro-segregation $[15,24,25]$. Most prior studies have relied on post-mortem microstructural observations that cannot determine the dynamics of solute transportation, heat flow and the topological evolution of the crystalline structure. Hence, it is very difficult to determine the exact mechanisms attributable to the application of magnetic fields on microstructural changes. In situ radiographic studies have proved more useful in this respect, demonstrating the effects of magnetic field-induced convection on solidification [13,26,27]. Recent advances in highspeed X-ray tomography enable time-resolved 3D observation of alloy solidification (known as 4D imaging - 3D space and time) [28-31], providing a useful tool to study solidification under magnetic fields. To make this possible, a suitable in situ rig is needed; a device described in this paper.

On the numerical side, the coupling of MHD flow with microscopic solidification requires a complex multi-physics approach, encompassing fluid flow, heat transfer, electromagnetic field modelling and solidification dynamics. Methods including phase field [32] and cellular automata (CA) [33] are being used to simulate solidification at the microstructural scale. Phase field [32] can be used to study microstructure growth at the microscopic scale. However, phase field is computationally expensive, preventing simulation of the coupled fluid flow and solidification occurring at the length scales relevant to our experiments. CA methods, on the other hand, allow large-scale microstructural simulation coupling solidification dynamics and fluid flow [34]. For this reason, the CA method has been chosen here to model the in situ experimental process so that both macro- and microscopic features can be represented.

In this study, we adopted an integrated experimental and numerical '4D' approach with synchrotron high-speed X-ray tomography and high-performance numerical modelling, enabling in situ observation and prediction of 3D structural and compositional evolution during solidification, with and without the magnetic fields. In terms of experiments, a bespoke temperature gradient stage (MagDS, Fig.1a) was built, which allows metal/alloy crystals to grow directionally (e.g. directional solidification). A homogeneous transverse magnetic field 
can be imposed on the sample using either a static permanent magnet pair producing $0.5 \mathrm{~T}$ or an electromagnet with a maximum strength of $1 \mathrm{~T}$ (Fig. $1 \mathrm{~b}$ and $1 \mathrm{c}$ ). MagDS has been integrated with synchrotron tomography at both the ID19 beamline of the European Synchrotron Radiation Facility (ESRF) and the I12 beamline of the UK Diamond Light Source (DLS) facility. We have used a method [35] that allows us to map the solute concentration during solidification, enabling 5D imaging (space, time and solute concentration). For the computational study, a parallelised numerical code called the 'ThermoElectric Solidification Algorithm' (TESA) was developed, coupling alloy solidification with fluid flow, driven by buoyancy and Lorentz force. The model is capable of simulating a large section of the experiment encompassing the entire diameter of the sample, capturing both micro- and meso-scopic scales. This integration of tools means that the experimental observation by 4D tomographic imaging can be used to validate the model and, in turn, the validated simulation code can support the correct interpretation of the experimental results. We show that with this approach, it is possible to determine the dominant mechanisms that cause the modulation of microstructures when a moderate, external, magnetic field is applied in various experimental and sample conditions, clearly demonstrating the significance of using magneto-hydrodynamics to control solidification microstructures.

\section{Methods}

\subsection{MagDS furnace}

The MagDS furnace includes mainly two parts: a small bespoke temperature gradient stage and strong magnetic fields (Fig. s1a). The temperature gradient stage $[28,35,36]$ is made of two heaters (both can reach $1000^{\circ} \mathrm{C}$ ) with a gap of $10 \mathrm{~mm}$ in between allowing X-ray to pass through (schematically shown in Fig. 1a). A proportional-integra-derivative controlling system is also developed allowing a constant temperature gradient to be kept within the gap when cooling the two heaters down. A $2 \mathrm{~mm}$ diameter cylindrical sample is placed inside the furnace in an alumina tube with inner diameter of $2 \mathrm{~mm}$. The magnetic fields can be produced by either permanent magnets (Fig. 1b) or electromagnets (Fig. 1C). For the permanent magnet, a permanent magnet pair (diameter of $80 \mathrm{~mm}$ ) with a gap of $50 \mathrm{~mm}$ are attached inside an iron yoke, producing a transverse homogeneous magnetic field of $0.5 \mathrm{~T}$ within the gap. For the electromagnet, an electromagnet made of water-cooled copper coils and iron cores (diameter of $50 \mathrm{~mm}$ ) was used. The opening between the two iron cores is $50 \mathrm{~mm}$. A homogeneous magnetic field adjustable and up to $1 \mathrm{~T}$ can be generated within the opening. The temperature gradient stage can be inserted inside the openings of permanent magnet pair or the electromagnet. Directional solidification of Al alloys was carried out via cooling the two heaters with a temperature difference at the same cooling rate.

\subsection{High-speed synchrotron $X$-ray tomography}

At the ID19 beam line of ESRF, high-speed X-ray tomography measurements were performed using a pink X-ray beam (peak energy at $31 \mathrm{keV}$ ). A high-resolution high-speed detector (Dimax) was used with a pixel size of $2.2 \mu \mathrm{m}$ and field of view (FOV) of $2.2 \times 2.2 \mathrm{~mm}$. A tomogram with 1000 projects was captured in $1 \mathrm{~s} .15 \mathrm{~s}$ was needed to download and transfer the data, hence the cycle time was $16 \mathrm{~s}$ [36].

At 112 beamline, the Diamond Light Source, a $53 \mathrm{keV}$ monochromatic X-ray beam (wavelength $0.023 \mathrm{~nm}$ ) is used. To acquire the X-ray tomogram, a PCO.edge camera was coupled with I12's camera module 3 [37], with $2560 \times 2160$ pixels, and a pixel size of $3.24 \mu \mathrm{m}$. The field of view was cropped to $1070 \times 1070$ pixels, corresponding to $3.467 \times 3.467 \mathrm{~mm}$. One tomogram 
consisted of 1000 projections over $180^{\circ}$ with an exposure time of $5 \mathrm{~ms}$ per projection, hence the total time per tomogram was $5 \mathrm{~s}$. After inclusion of additional sample rotation and data download, the cycle time was $10 \mathrm{~s}$.

\subsection{Tomographic data analysis}

3D image processing and quantification was performed using Avizo 9.4 (ThermoFisher Scientific) and ImageJ (US NIH, Bethesda, MD, USA). The tomogram was first filtered using an anisotropy diffusion filter, followed by a background correction. Then the solid phase was segmented using Otsu's threshold method [38]. The $\mathrm{Cu}$ concentration mapping was performed based on the fact that the grey value of the X-ray tomographic images at each pixel is a linear function of density of the material at that pixel. Procedures for composition mapping can be found in [35].

\subsection{Numerical modelling}

The numerical results were produced using a bespoke 'ThermoElectric Solidification Algorithm' (TESA) code that couples solidification, fluid flow and electromagnetism (including thermoelectric effects). The code incorporates three solvers, one for each aspect. Solidification uses a decentred octahedral cellular automaton method based on the $\mu$ matIC code [39]. Staggered finite difference methods are used to solve Navier-Stokes' equations and Ohm's law. The computational domain is partitioned spatially into equal sized sub-domains that run on separate processor cores with inter-processor communications, handled by Message Passing Interface, passing local boundary conditions. Using a 160 core Intel Xeon cluster, this allowed for a grid resolution on the microscale to capture the spatial and temporal scales of the experiments within a reasonable time frame of several days.

Solidification is predicted using a cellular automata method [33] where the temporal change in solid fraction, $f_{s}$, is given by

$$
\frac{\partial f_{s}}{\partial t}=\frac{1}{C_{l}(1-k)}\left(-\frac{\partial C_{e}}{\partial t}+\left(1-(1-k) f_{s}\right) \frac{\partial C_{l}}{\partial t}\right)
$$

where $C_{l}$ (wt.\%) is the liquid solute concentration, $C_{e}$ (wt.\%) is the equivalent concentration and $k$ is the partitioning coefficient.

MHD are governed by the Lorentz force, $\mathbf{j} \times \mathbf{B}$, where $\mathbf{j}$ is the current density and $\mathbf{B}$ the magnetic field. The current density can be described by a generalised form of Ohm's law

$$
\mathbf{j}=\sigma(\mathbf{E}-S \nabla T+\mathbf{u} \times \mathbf{B})
$$

where $\sigma\left(\mathrm{S} \mathrm{m}^{-1}\right)$ is the electrical conductivity, $\mathbf{E}\left(\mathrm{V} \mathrm{m}^{-1}\right)$ the electric field, $S\left(\mathrm{~V} \mathrm{~K}^{-1}\right)$ the Seebeck coefficient and $\mathbf{u}\left(\mathrm{m} \mathrm{s}^{-1}\right)$ the velocity. The term $S \nabla T$ is responsible for thermoelectric currents, which drive TEMHD, while the induced currents, $\mathbf{u} \times \mathbf{B}$, give rise to flow driven by $F_{E M}$ including electromagnetic damping and electromagnetic stirring.

Due to the short length scales and relatively slow fluid velocities investigated, the Reynolds number is small and momentum balance can be described by incompressible Stoke's flow given as

$$
\rho \frac{\partial \mathbf{u}}{\partial t}=-\nabla p+\mu \nabla^{2} \mathbf{u}+\mathbf{j} \times \mathbf{B}+\rho \mathbf{g} \beta\left(C_{l}-C_{\mathrm{ref}}\right)
$$


where $\rho\left(\mathrm{kg} \mathrm{m}^{-3}\right)$ is the density, $p(\mathrm{~Pa})$ is the pressure, $\mu$ (Pa s) is the dynamic viscosity. The final term accounts for the solute buoyancy force, where $\mathbf{g}\left(\mathrm{m} \mathrm{s}^{-2}\right)$ is the acceleration due to gravity, $\beta\left(w t . \%^{-1}\right)$ is the solute expansion coefficient and $C_{\text {ref }}$ (wt.\%) is a reference concentration. Convective transport of solute is governed by

$$
\frac{\partial \mathrm{C}_{e}}{\partial t}=\nabla \cdot\left(D \nabla C_{l}\right)-\mathbf{u} \cdot \nabla C_{l}
$$

where $D\left(\mathrm{~m}^{2} \mathrm{~s}^{-1}\right)$ is the mass diffusivity and finally continuity of charge and continuity of mass gives

$$
\nabla \cdot \mathbf{j}=\nabla \cdot \mathbf{u}=0
$$

\section{Results and discussion}

In this section, the interaction and competition between TEMHD and EMS during metal solidification was demonstrated through three case studies under different conditions, with both experimental and numerical results.

\section{Case 1. Control Case - Formation of tilted interface by TEMHD}

A tilted interface during directional solidification under a transversal magnetic field has been observed experimentally by post-mortem characterization [22,25], and TEMHD has been proposed as the main mechanism [22,25,40]. Here, based on previous experiments [22,25], we designed a quasi in situ experiment to confirm the existence of TEMHD during alloy solidification and to validate the numerical model. During the experiment, an Al15Cu (weight percent) sample was first fully melted in a temperature gradient $(\nabla T=10 \mathrm{~K} / \mathrm{mm})$, then, solidified by cooling down both heaters of the temperature gradient stage at the same cooling rate $(\mathrm{CR})$ of $0.05 \mathrm{~K} / \mathrm{s}$. During the process, the sample was kept stationary without rotation. When the solid phase grew to approximately half the height of the field of view, the cooling process was stopped. Hence the dendrites stopped growing. Then the temperature of both heaters was held constant for 10 minutes, which was used to establish quasi-steady equilibrium conditions [41]. A tomogram (captured in $5 \mathrm{~s}$ ) was taken after the holding period. The morphology of the solid phase after image segmentation is shown in Fig. 2a, demonstrating a relatively flat and levelled solid/liquid interface. Note that the dendritic structure coarsened slightly during holding, making the separation of different dendrites difficult during image processing, hence the dendrite structure in Fig. 2a appears to be a connected network. Afterwards, a $0.5 \mathrm{~T}$ transverse magnetic field was applied using the electromagnet. After holding for another 10 minutes under the same temperatures and magnetic field, a second tomogram was taken (the magnet was off when taking the tomogram). As shown in Fig. 2b, the solid-liquid interface became tilted.

This experiment was specifically designed to show the existence of TEMHD effects during solidification. After holding the semi-solid sample for $10 \mathrm{~min}$ at temperature, a quasi-steady state equilibrium is reached where solute and buoyancy flow can be assumed to be diminished. Under such a steady state condition, a flat and levelled solid/liquid interface was formed. In the Supplementary Note, we have shown that (1) the under steady state condition, the solute and buoyancy flow was diminished; (2) when rotating the sample for taking the tomogram, the effect of 'spin up' velocity can also be neglected; (3) during rotation for 
tomogram, the centrifugal force $F_{C F}$ is less than $10 \mathrm{~N} / \mathrm{m}^{3}$, much smaller than the thermoelectric force which is of $O\left(10^{4} \mathrm{~N} / \mathrm{m}^{3}\right)$, and the flow velocity induced by centrifugal force would only be a maximum of $1 \mu \mathrm{m} / \mathrm{s}$, hence is negligible. As the flow is diminished, the modulation of flow by $F_{E M}$ (such as electromagnetic damping effect) can then be mostly ignored. The observation of a tilted interface morphology when a static magnetic field was applied for another $10 \mathrm{~min}$ may be solely attributed to TEMHD, confirming results made by others $[22,25]$. In order to show that this is the case, and to reveal the underlying mechanisms and dynamics of the tilted interface formation, we performed simulations using TESA under similar conditions to our experiments.

The numerical simulation uses a Seebeck power, $S=10^{-6} \mathrm{~V} / \mathrm{K}$, characteristic of Al15Cu [40]. The results are shown in Fig. $2 c$ and $2 d$. After reaching a quasi-steady state equilibrium (holding for $10 \mathrm{~min}$ ), the interface with $B=0 \mathrm{~T}$ is essentially flat and levelled (Fig. 2c). And the simulated dendritic structure is closely similar to the experimental observation (Fig.2a). When a magnetic field of $B=0.5 \mathrm{~T}$ was then imposed, the interface became tilted (Fig. $2 \mathrm{~d}$ ), showing an excellent match to the experimental observation, providing strong evidence that the TEMHD effect is the driving force for this phenomenon. In the absence of a magnetic field, there is only a single source of fluid flow during solidification, natural convection, due to the higher density of $\mathrm{Cu}$ than Al. However, as the system reaches quasi equilibrium after the sample was held for $10 \mathrm{~min}$, natural convection diminishes as the gravitational force in the liquid is balanced by a vertical hydrostatic pressure gradient (Fig. 2f) and flat isosurfaces of solute concentration form, which will lead to the formation of a levelled solid liquid interface. In the presence of a magnetic field, there is the addition of the thermoelectric Lorentz force $\left(F_{T E}\right)$, which is perpendicular to both the gravitational force and the magnetic field direction (schematically shown in Fig. 2f). When the magnetic field is turned on, the forces are not balanced, and the Lorentz force accelerates flow through the inter-dendritic region and up the sample wall (Flow 1 and 2 in Fig. 2e). At this location, convective transport of high solute concentration up the microstructure causes remelting (Fig. 2e, the right-hand side). Conservation of mass causes flow (Flow 3 in Fig.2e) to enter the inter-dendritic region on the other side of the sample (Fig. 2e, the left-hand side), which promotes solidification. Consequently, the interface takes a tilted shape. At equilibrium, high pressure forms in the melted region and a low pressure in the solidified region, thus the pressure gradient serves to balance both the Lorentz and gravitational forces (Fig.2f).

In summary, this first case verifies that TEMHD can alter solidification microstructures, supported by both quasi in situ synchrotron imaging and advanced numerical simulation. The results show that with an application of a static magnetic field to a directionally solidifying alloy, the TEMHD effect drives fluid flow that circulates around the solid-liquid interface perpendicular to the magnetic field, resulted in macro-segregation and a tilted solid-liquid interface. The experimental results are consistent with findings from the literature $[22,25]$ and serve as a benchmark validation case for the numerical TESA model. The quasi in situ experiment allows us to capture snapshots of the microstructural evolution at critical time points, showing how the interface without magnetic field became flat as a result of holding, and how that was tilted as a result of the application of magnetic field. The simulation, replicating the experimental conditions, provided not only the morphological evolution as seen in the experiment, but also the solute flow during the process, allowing a physical understanding of the tilted interface formation. The transient evolution dynamics are given in Supplementary Video 1. This case also demonstrated that our numerical model is capable 
of handling complicated physics involving fluid flow, solidification and magnetism, to dynamically predict the interface which is not defined a priori.

\section{Case 2. Formation of Archimedes screw structure}

In the second set of experiments, the $\mathrm{Al} 15 \mathrm{Cu}$ alloy was solidified under $|\mathrm{B}|=0.5 \mathrm{~T}$ and $\nabla T=$ $10 \mathrm{~K} / \mathrm{mm}$ via cooling down both heaters of the MagDS furnace at CR of $0.02 \mathrm{~K} / \mathrm{s}$. During solidification, the sample was continuously rotating at $0.5 \mathrm{~Hz}$ and high-speed tomograms were captured in $1 \mathrm{~s}$ during the process every $16 \mathrm{~s}$. An unusual screw-like microstructure was observed as shown in Figure 3a-3c, where the green and blue contours show the primary $\alpha$ Al phase and the red contour shows the highly enriched Cu eutectic. The eutectic region was clearly distributed around the edge of the sample, forming a helix. The screw structure can also be altered via different experimental conditions such as cooling rates and thermal gradients (Fig. 4). This result is in distinct contrast to the microstructure formed without an imposed magnet field as shown in Figs. $3 \mathrm{~d}$ to $3 \mathrm{f}$, where the eutectic-localized region was positioned at one side of the sample.

The screw structure shows striking similarities to the observations of Kaldre et al. [43], where a helical segregation pattern was observed in a $\mathrm{Sn} 10 \mathrm{~Pb}$ alloy directionally solidified in a rotating transverse magnetic field of $0.5 \mathrm{~T}$ at rotation periods of $300 \mathrm{~s}$ and $600 \mathrm{~s}$. Their analysis showed that the separation distance between each turn of the helix was equal to the product of the rotation period and growth velocity. They estimated the effect of fluid flow introduced from the time varying magnetic field and showed that at such slow rotation speeds, the electromagnetic stirring (EMS) effect was small. At faster rotation (shorter rotation periods ca. $150 \mathrm{~s}$ ) the screw structure did not appear. They concluded that the macrosegregation from TEMHD was responsible for the screw structure, i.e. that a slowly rotating tilted interface from Case 1 had formed. At a faster rotation speed (e.g. with a rotation period of 60 and $90 \mathrm{~s}$ ) no screw structure was formed. However, in our work with a much faster rotation (e.g. a rotation period of 1s), a well-defined screw structure is clearly observed. The thread spacing does not follow the same trend as the TEMHD mechanism proposed by Kaldre [43], implying that a second regime for the formation of screw structure has been discovered, along with an entirely different mechanism.

To understand the formation process of this screw structure, the Cu solute concentration was mapped from the acquired 4D data using the method as shown in Ref [35]. Fig.5a to 5e show a vertical slice at the middle of the solidifying sample, at different time steps without the application of magnetic field $(|\mathbf{B}|=0 T)$. As the temperature started cooling from the liquid state, the primary dendrites grew upwards. Due to a slight horizontal temperature gradient, the dendrites grew at one side of the sample [35]. Cu was rejected into the liquid on the other side of the sample. As $\mathrm{Cu}$ is heavier than $\mathrm{Al}$, the segregated $\mathrm{Cu}$ dropped down, forming a macro-segregation zone at the other side of the sample (Supplementary video 2 and 3). With the application of the magnetic field, as shown in Fig $5 f$ to $5 \mathrm{~g}$, the dendrites formed first at the central core (Fig $5 \mathrm{~g}$ ). In the liquid, ahead of the solid-liquid interface, the Cu solute, after being rejected from the growing primary phase, flew upwards like a plume (Figs. $5 \mathrm{~g}$, $5 \mathrm{~h}$ and Supplementary video 4 and 5). This led to a dome-shape $\mathrm{Cu}$-concentrated region in the liquid with a high concentration of Cu solute. In the centre of the sample, Cu solute was depleted and the primary solid grew extensively in this region first. This observation is quite unusual as 
it implies there is an additional convective transport mechanism that overcomes the solutedriven buoyancy flow, driving the upward flow of denser $\mathrm{Cu}$-enriched melt.

Fig. $5 \mathrm{k}$ to 50 show the growth of the primary phase of several successive tomograms. Two well-grown dendritic branches observed at $640 \mathrm{~s}$ (Fig.5k), were later remelted entirely at 688 $\mathrm{s}$ (Fig.5I). This observation implies that a flush of $\mathrm{Cu}$-enriched hot liquid flew to the region, remelting the root of the dendrites, which then detached from the main structure, flew upwards into the hot liquid and completely melted. The detachment and flowing of dendrites can be observed frequently from the Supplementary video 4. In Fig. 5I, a small side branch indicated by the red arrow survived from the remelting process and grew towards the left side of the sample, developing into a mature dendrite at $720 \mathrm{~s}$ (Fig.5m). The side branches of this new dendrite grew downwards, then partially remelted (Fig.5n). After this turbulent growth of the primary phase (remelting, detachment, downward growth and further remelting), a round-shaped channel, free of the primary dendrites, was formed around the sample periphery (Fig.5o, circled). From the composition mapping analysis, this channel was enriched with $\mathrm{Cu}$ solute. This process was repeated as solidification continued and led to the formation of the helical channel (Supplementary video 6). Fig. $5 p$ shows the quantification of the primary phase including the foremost tip position and volume fraction. There is erratic localized growth and remelting, indicated by the change of tip position of the foremost dendrite; however, the volume-averaged solid fraction as a function of time is linear.

As shown, many phenomena were observed while the sample was rotating in a magnetic field. These include: (1) the formation of a screw structure with a rounded helical channel enriched with the Cu solute; (2) solidification of the solid core before the formation of channels, (3) a radial dependence of $\mathrm{Cu}$ concentration in the solid, (4) fragmentation of dendrite, then (5) convective transport of the dendrite fragments and (6) the formation of $\mathrm{Cu}$ rich plumes.

Understanding the driving force responsible for these effects is not immediately clear. When the solidifying sample rotates within the magnetic field, flows can be driven by both $F_{E M}$ and $F_{T E}$. In the liquid ahead of the mushy zone, the Hartmann number $\mathrm{Ha}=|\mathbf{B}| L(\sigma / \mu)^{1 / 2}=18$ ( $L$ is the characteristic length scale, $\mu$ is dynamic viscosity), indicating strong $F_{E M}$. However, in the inter-dendritic region of the mushy zone, $\mathrm{Ha}=0.2$, indicating that $F_{E M}$ is small and TEMHD will be relatively unaffected. The segregation of solute from TEMHD will always be directed perpendicular to the static magnetic field (as demonstrated by Case 1), and with the sample rotating, the segregated $\mathrm{Cu}$ is transported with the rotation. Analogous to the Coriolis effect, a helical flow of $\mathrm{Cu}$ solute would be expected. On the other hand, due to the electromagnetic force and sample rotating, a second relative flow can also be introduced. When solidifying samples are rotating (without a magnetic field), both the liquid and solid can be described to be in solid-body rotation, such that in the frame of reference of the solid phase, there is no apparent fluid flow generated by sample rotation. However, when a magnetic field is applied, the liquid flow slows down due to electromagnetic damping, while the solid ( $\alpha$-Al dendrites) still rotates with the same angular velocity as the rotation stage. Consequently, in the frame of the solid phase, there is an apparent rotational flow as the solid is mechanically driven through the slower moving liquid. Due to Galilean invariance between the frame of the magnetic field and sample frame this is similar to flow introduced by rotating the magnetic field around a static sample, hence similarly electromagnetic stirring (EMS) was used to describe the process. 
Determining which effect (TEMHD or EMS) dominates and is responsible for the experimentally observed screw structure was not immediately evident. Using the computational TESA model, the electromagnetic Lorentz force and thermoelectric magnetic force can be decoupled, isolating each phenomenon separately. This can be achieved by either assuming zero Seebeck power $(S=0)$ so that thermoelectric magnetic forces are removed, or by turning off the electromagnetic forces in the TESA simulation.

Fig.6a shows the numerical results where only TEMHD is considered (Seebeck power $S=10^{-6}$ $\mathrm{V} / \mathrm{K}$ ) while turning off electromagnetic forces. The result shows that the flow from TEMHD pushes $\mathrm{Cu}$-enriched liquid to the edge of the sample, but primarily only the core solidifies. The distinct screw structure observed by experiments (Fig. 3b) is not predicted. In contrast, when only electromagnetic force is considered (setting Seebeck power $S=0$ in the model), the numerical model produces a screw structure (Fig.6b) similar to the experiment (Fig. 3b). Results for the fully coupled simulations, including both electromagnetic Lorentz force $\left(S=10^{-}\right.$ ${ }^{6} \mathrm{~V} / \mathrm{K}$ ) and thermoelectric magnetic force, are given in Fig.6c and show that the screw structure still forms, but with the introduction of TEMHD the screw is slightly less well defined compared to Fig. 6b. Unlike the slowly rotating results of Kaldre et al. [43] where TEMHD was dominant, these findings suggest that the mechanism leading to the screw structure for samples rotating quickly in a magnetic field is EMS; the introduction of TEMHD only causes a minor modulation of the screw structure under these conditions. The simplest explanation for the formation of the screw structure is attributed to $F_{E M}$, driving an angular flow velocity, causing magnetic stirring effect, similar to a rotating magnet field, while $F_{T E}$ drives a rotating radial velocity. Essentially at a faster rotation the time averaged flow field of EMS is relatively unchanged, while for TEMHD, the time-averaged flow diminishes.

With the numerical result, the experimentally observed phenomena can be explained. Assuming nucleated dendrites are formed when $|\mathbf{B}|=0 \mathrm{~T}$ within a temperature gradient, as the sample is rotating and a transverse static magnetic field of $0.5 \mathrm{~T}$ is applied, the liquid flow velocity is reduced due to the electromagnetic damping. The fluid is then pushed through by the rotating dendrites whose velocity is not influenced by the magnetic field. At the outer radius of the sample with a high angular velocity, the force on the liquid is higher than at the centre, generating a rotational flow (Fig. 5e), similar to electromagnetic stirring effect. Some of the flow is forced up ahead of the solid-liquid interface, remelting the primary phase and forming the first exit of the helical channel (Fig. 5d). In the centre, the force is lower and to preserve continuity the flow will be downward (Fig. 5e), leading to the solidification of the primary phase in the core. The downward flow will also begin feeding and stabilising the channel.

When the solid phase forms a screw with helical channels, there is a direct analogy to flow generated by an Archimedes screw, where the rotation of the solid screw generates a vertical pressure gradient, forcing liquid flow up the helical channel. This upwards flow might be the reason for the formation of the Cu-solute plume observed by the experiment (Fig. $5 \mathrm{~g}$ ). As liquid flows up the channel, continuity requires that any flow leaving the top of the screw must be replaced. However, lower down the sample, the sample must be fully solidified, and becomes unable to feed the flow up the helix. In this case, the liquid flow to feed the channel through the sample core which solidified first and is porous with inter dendritic liquid. Near the tip of the screw, the fluid, which is forced out of the helical channel into the bulk, is replaced by the inter-dendritic fluid from the core, which in turn is replaced by an incident flow from the bulk into the core. This forms a radial-vertical fluid circulation as shown in Fig. 
$6 e$ and $6 f$ at the tip of the screw. Further down the sample, where the core inter-dendritic space is smaller due to coarsening, flow is exchanged through the gaps between successive turns of the helical channel. The liquid flow process is also highlighted in Fig. $6 \mathrm{~g}$ where the red and blue isosurfaces represent upward and downward flow respectively. The downward flow is concentrated in the gaps in the thread of the screw, while the upward flow follows the channel. The feeding incident flow, focussed on the centre of the sample, has a low $\mathrm{Cu}$ concentration and is the primary reason why the core solidifies first. As the core solidifies first it also solidifies at a higher temperature and at a lower $\mathrm{Cu}$ concentration, capturing less $\mathrm{Cu}$ in the primary phase. As the thread forms later, more $\mathrm{Cu}$ is captured leading to an increase in $\mathrm{Cu}$ concentration in the thread. This was observed by both experiment (Fig. 5j)) and the simulation (Fig. 6h). A video showing the numerical prediction of the evolution of the screw structure is given in Supplementary video 7.

The circulation of high $\mathrm{Cu}$ concentration fluid is also the cause for dendrite fragmentation. Since the core solidifies ahead of the sample edge, primary and secondary dendrite arms can grow over the channel. With the formation of helical channel, $\mathrm{Cu}$ solute continuously accumulated within the channel as shown in Fig $5 \mathrm{~h}-5 \mathrm{j}$. The high $\mathrm{Cu}$ concentrated liquid remelts the root of these dendrite arms causing them to detach. Many detachments were observed at high frequency (Supplementary video 4), indicating that the rotation flow is very strong and disruptive. The broken off fragments can be distributed in the melt and offer sites for grain nucleation [10], a possible reason for columnar-to-equiaxed transition during directional solidification under an alternating magnetic field [21]. It is noted that the detachment and flow-up of the dendrite branches are not implemented in the model, as modelling the translation of grains accurately is currently beyond the capability of the model. However, with such large density differences between the solid and liquid, the floating fragments would introduce a strong suction force in their wake, amplifying the plumes of ejected $\mathrm{Cu}$ at the exit of the helical channel.

In summary, through a joint experimental and numerical investigation, a second regime for the formation of a screw like structure has been found at fast rotations under a transversal magnetic field, where the liquid flow became much more disrupted, compared to the sample solidified without the magnet. The experimental observations showed many phenomena that are both predicted and explained by the numerical model. By decoupling the $F_{E M}$ and $F_{T E}$ induced flow in the numerical model, we show that for fast rotation, the formation of the screw structure can be attributed to $F_{E M}$ induced melt stirring.

\section{Case 3. Structure refinement}

Cases 1 and 2 demonstrated the dominance of TEMHD and EMS respectively. The third case presented here explores the phenomena arising from an increase in the TEMHD effect with a fast-rotating sample.

To investigate this, a tenfold increase in the Seebeck power as compared to Case 2 was simulated (i.e. $S=10^{-5} \mathrm{~V} / \mathrm{K}$ ), increasing the TEMHD induced flow to a level comparable to the EMF flow. As shown in Fig.7a and 7b, the screw structure is not present. Instead, a refined microstructure with long needle-like cells and a flat and levelled solid-liquid interface forms. In this case, due to the high Seebeck power, TEMHD velocities are higher, and mass transport due to TEMHD becomes more important than Case 2. As a result, the solute is pushed up and concentrated ahead of the interface (Fig. 7b). Therefore, a homogenised high concentration 
solute layer above the interface is formed. Solidification then occurs at a much higher concentration, and lower temperature. Under these conditions, the structure becomes significantly refined and radial macro-segregation is absent, which are both beneficial microstructural features. This microstructure is significantly different from those with only TEMHD (Fig. 6a) or EMS (Fig. 6b), therefore, indicating that it is the coupling of TEMHD and EMS that drives the refinement of primary arm spacing. A video of the microstructure evolution is given in Supplementary Video 8.

To validate the numerical prediction, we performed in situ synchrotron experiments on an Al5.5Si3.5Cu based alloy, which is expected to have much higher Seebeck coefficient than Al15Cu due to the semiconductor-like properties of Si. Fig. 7c and 7d show experimental results with $|\mathbf{B}|=0 \mathrm{~T}$, where a large dendritic structure is observed together with a macrosegregation region similar to $\mathrm{Al} 15 \mathrm{Cu}$. Fig.7g and $7 \mathrm{~h}$ show experimental results with $|\mathbf{B}|$ $=0.5 \mathrm{~T}$. A significantly refined dendritic structure with much smaller primary dendrite arm spacing is observed, which compares favourably with the numerical results (Fig. 7a and 7b). Refinement of dendrite arm spacing during directional solidification under a static transversal magnetic field was observed in $\mathrm{Fe}-\mathrm{Ni}$ and $\mathrm{Pb}-\mathrm{Bi}$ alloys [19]. Refinement of $\mathrm{Al}_{2} \mathrm{Cu}$ phase in hypereutectic $\mathrm{Al}-\mathrm{Cu}$ alloy was observed during directional solidification under axial magnetic fields [3]. In those works, TEMHD alone was attributed to be the main mechanism for the observed refinement. However, in our study, we have shown that the coupled effect of TEMHD and EMS was responsible to produce the fine microstructures where the sample was rotating fast in a static magnetic field.

This case has shown that when EMS and TEMHD flows are comparable, the microstructure can be significantly refined. Hence, a favourable microstructural design is possible when utilising MHD in solidification. The interplay between EMS and TEMHD is a key factor and therefore it may be critical to precisely measure the Seebeck coefficient, perhaps build on techniques such as those used in [19] when designing new processes.

\section{Conclusions}

By combining high performance computational simulation and synchrotron X-ray imaging, the microstructural modification arising from MHD during alloy solidification was revealed. The following conclusions can be made:

1. An in situ solidification rig, enabling strong magnetic fields to be applied, was developed that allowed high speed synchrotron tomographic visualization of solidifying micro- and macro- structure under the influence of magnetic fields.

2. A powerful numerical simulation code was developed coupling MHD to interface dynamics, allowing for the various solidification microstructural features to be predicted, and the corresponding underlying physical mechanisms to be revealed.

3. After holding a directionally solidifying Al15Cu sample in a semi-solid state, a flat interface between the mushy zone and the liquid was formed. When a static transverse magnetic field of $0.5 \mathrm{~T}$ was applied, a tilted interface and a highly macrosegregated region was formed, which was attributed to the fluid and solute flow driven by TEMHD. This benchmark case was used to validate the simulation, showing that the model can predict the change of solidifying microstructure as a result of MHD.

4. When a rotating Al15Cu alloy was directionally solidified under a transverse magnetic field, an Archimedes' screw-like structure was formed. Our simulation demonstrates that magnetic stirring rather than TEMHD was responsible for this fast rotation case. 
Additionally, both our experimental and simulation results demonstrate that under similar conditions, but for an alloy with a higher Seebeck coefficient, directionally solidified dendrites with highly refined primary arm spacing can be produced.

5. Novel approaches for microstructure control can be designed using MHD offering innovative potential applications. The macro segregation due to the TEMHD effect could be developed into an industrial technique for materials refinement and recycling. At large scales, the helical channel has the potential to mitigate hot tearing in ingots, as the melt is forced to flow into these channels and provide a means to repair the initiation of any tear close to the surface. The refinement of the primary dendrite arm spacing reduces the microsegregation length scale, refining secondary phases and improving mechanical properties. This is true for both for components used with the as cast structure, such as $\mathrm{Ni}$-based single crystal turbine blades, and when subsequent thermomechanical processing is applied.

\section{Reference}

[1] L. Zhang, S. Wang, A. Dong, J. Gao, L.N.W. Damoah, Application of Electromagnetic (EM) Separation Technology to Metal Refining Processes: A Review, Metall. Mater. Trans. B. 45 (2014) 2153-2185. https://doi.org/10.1007/s11663-014-0123-y.

[2] H.A. Chedzey, D.T.J. Hurle, Avoidance of Growth-striae in Semiconductor and Metal Crystals grown by Zone-melting Techniques, Nature. 209 (1966) 1246-1248.

[3] J. Wang, S. Yue, Y. Fautrelle, P.D. Lee, X. Li, Y. Zhong, Z. Ren, Refinement and growth enhancement of Al2Cu phase during magnetic field assisting directional solidification of hypereutectic Al-Cu alloy, Sci. Rep. 6 (2016) 24585.

https://doi.org/10.1038/srep24585.

[4] P. de Rango, M. Lees, P. Lejay, A. Sulpice, R. Tournier, M. Ingold, P. Germi, M. Pernet, Texturing of magnetic materials at high temperature by solidification in a magnetic field, Nature. 349 (1991) 770-772. https://doi.org/10.1038/349770a0.

[5] R.W. Series, D.T.J. Hurle, The use of magnetic fields in semiconductor crystal growth, J. Cryst. Growth. 113 (1991) 305-328. https://doi.org/10.1016/0022-0248(91)900365.

[6] I. Kaldre, A. Bojarevičs, I. Grants, T. Beinerts, M. Kalvāns, M. Milgrāvis, G. Gerbeth, Nanoparticle dispersion in liquid metals by electromagnetically induced acoustic cavitation, Acta Mater. 118 (2016) 253-259.

https://doi.org/10.1016/j.actamat.2016.07.045.

[7] N. Kang, H. Yuan, P. Coddet, Z. Ren, C. Bernage, H. Liao, C. Coddet, On the texture, phase and tensile properties of commercially pure Ti produced via selective laser melting assisted by static magnetic field, Mater. Sci. Eng. C. 70 (2017) 405-407. https://doi.org/10.1016/j.msec.2016.09.011.

[8] C. Vives, C. Perry, Effects of magnetically damped convection during the controlled solidification of metals and alloys, Int. J. Heat Mass Transf. 30 (1987) 479-496. https://doi.org/10.1016/0017-9310(87)90263-8.

[9] Z. Yan, M. Chen, Y. Teng, J. Yang, L. Yang, H. Gao, Forced Flow and Solidification Process of $\mathrm{Sn}-3.5 \% \mathrm{~Pb}$ Melt in Hollow Billet Under Rotating Magnetic Field, J. Mater. 
Eng. Perform. 24 (2015) 1059-1064. https://doi.org/10.1007/s11665-014-1303-2.

[10] Z. Yan, X. Li, Z. Cao, X. Zhang, T. Li, Grain refinement of horizontal continuous casting of the CuNi10Fe1Mn alloy hollow billets by rotating magnetic field (RMF), Mater. Lett. 62 (2008) 4389-4392. https://doi.org/10.1016/j.matlet.2008.07.010.

[11] J.A. Shercliff, Thermoelectric magnetohydrodynamics, J. Fluid Mech. 91 (1979) 231. https://doi.org/10.1017/S0022112079000136.

[12] S.N. Tewari, R. Shah, H. Song, Effect of Magnetic-Field on the Microstructure and Macrosegregation in Directionally Solidified Pb-Sn Alloys, Met. Mater. Trans. A. 25A (1994) 1535.

[13] Y. Fautrelle, J. Wang, G. Salloum-Abou-Jaoude, L. Abou-Khalil, G. Reinhart, X. Li, Z.M. Ren, H. Nguyen-Thi, Thermo-Electric-Magnetic Hydrodynamics in Solidification: In Situ Observations and Theory, Jom. 70 (2018) 764-771. https://doi.org/10.1007/s11837018-2777-4.

[14] X. Li, Z. Ren, Y. Fautrelle, Effect of a high axial magnetic field on the microstructure in a directionally solidified Al-Al2Cu eutectic alloy, Acta Mater. 54 (2006) 5349-5360. https://doi.org/10.1016/j.actamat.2006.06.051.

[15] R. Moreau, O. Laskar, M. Tanaka, D. Camel, Thermoelectric magnetohydrodynamic effects on solidification of metallic alloys in the dendritic regime, Mater. Sci. Eng. A. 173 (1993) 93-100. https://doi.org/10.1016/0921-5093(93)90194-J.

[16] Y.Y. Khine, J.S. Walker, F.R. Szofran, Thermoelectric magnetohydrodynamic flow during crystal growth with a moderate or weak magnetic field, J. Cryst. Growth. 212 (2000) 584-596. https://doi.org/10.1016/S0022-0248(00)00209-8.

[17] A. Kao, P.D. Lee, K. Pericleous, Influence of a Slow Rotating Magnetic Field in Thermoelectric Magnetohydrodynamic Processing of Alloys, ISIJ Int. 54 (2014) 12831287.

[18] I. Kaldre, Y. Fautrelle, J. Etay, A. Bojarevics, L. Buligins, Thermoelectric current and magnetic field interaction influence on the structure of directionally solidified Sn-10 wt.\%Pb alloy, J. Alloys Compd. 571 (2013) 50-55. https://doi.org/10.1016/j.jallcom.2013.03.211.

[19] X. Li, Z. Lu, Y. Fautrelle, A. Gagnoud, R. Moreau, Z. Ren, Effect of a weak transverse magnetic field on the microstructure in directionally solidified peritectic alloys, Sci. Rep. 6 (2016) 37872. https://doi.org/10.1038/srep37872 (2016).

[20] X. Li, A. Gagnoud, Y. Fautrelle, Z. Ren, R. Moreau, Y. Zhang, C. Esling, Dendrite fragmentation and columnar-to-equiaxed transition during directional solidification at lower growth speed under a strong magnetic field, Acta Mater. 60 (2012) 3321-3332. https://doi.org/10.1016/j.actamat.2012.02.019.

[21] X. Li, Y. Fautrelle, K. Zaidat, A. Gagnoud, Z. Ren, R. Moreau, Y. Zhang, C. Esling, Columnar-to-equiaxed transitions in al-based alloys during directional solidification under a high magnetic field, J. Cryst. Growth. 312 (2010) 267-272. https://doi.org/10.1016/j.jcrysgro.2009.10.002.

[22] J. Wang, Z. Ren, Y. Fautrelle, X. Li, H. Nguyen-Thi, N. Mangelinck-Noel, G.S.A. Jaoude, Y. Zhong, I. Kaldre, A. Bojarevics, Modification of liquid/solid interface shape in 
directionally solidifying Al-Cu alloys by a transverse magnetic field, J. Mater. Sci. 48 (2013) 213-219. https://doi.org/10.1007/s10853-012-6730-6.

[23] X. Li, Y. Fautrelle, Z. Ren, Influence of an axial high magnetic field on the liquid-solid transformation in Al-Cu hypoeutectic alloys and on the microstructure of the solid, Acta Mater. 55 (2007) 1377-1386. https://doi.org/10.1016/j.actamat.2006.10.007.

[24] S.N. Tewari, R. Shah, H. Song, Effect of magnetic field on the microstructure and macrosegregation in directionally solidified Pb-Sn alloys, Metall. Mater. Trans. A. 25 (1994) 1535-1544. https://doi.org/10.1007/BF02665485.

[25] X. Li, Y. Fautrelle, Z. Ren, R. Moreau, Formation mechanism of axial macrosegregation of primary phases induced by a static magnetic field during directional solidification, Sci. Rep. 7 (2017) 1-13. https://doi.org/10.1038/srep45834.

[26] G. Salloum-Abou-Jaoude, J. Wang, L. Abou-Khalil, G. Reinhart, Z. Ren, N. MangelinckNoel, X. Li, Y. Fautrelle, H. Nguyen-Thi, Motion of equiaxed grains during directional solidification under static magnetic field, J. Cryst. Growth. (2014) 1-6. https://doi.org/10.1016/j.jcrysgro.2014.10.058.

[27] G. Salloum-Abou-Jaoude, J. Wang, L. Abou-Khalil, G. Reinhart, Z. Ren, N. MangelinckNoel, X. Li, Y. Fautrelle, H. Nguyen-Thi, Motion of equiaxed grains during directional solidification under static magnetic field, J. Cryst. Growth. 417 (2015) 25-30. https://doi.org/10.1016/j.jcrysgro.2014.10.058.

[28] B. Cai, J. Wang, A. Kao, K. Pericleous, A.B. Phillion, R.C. Atwood, P.D. Lee, 4D synchrotron X-ray tomographic quantification of the transition from cellular to dendrite growth during directional solidification, Acta Mater. 117 (2016) 160-169. https://doi.org/10.1016/j.actamat.2016.07.002.

[29] L.K. Aagesen, A.E. Johnson, J.L. Fife, P.W. Voorhees, M.J. Miksis, S.O. Poulsen, E.M. Lauridsen, F. Marone, M. Stampanoni, Universality and self-similarity in pinch-off of rods by bulk diffusion, Nat. Phys. 6 (2010) 796-800. https://doi.org/10.1038/nphys1737.

[30] D. Kammer, P.W. Voorhees, The morphological evolution of dendritic microstructures during coarsening, Acta Mater. 54 (2006) 1549-1558. https://doi.org/10.1016/j.actamat.2005.11.031.

[31] B. Cai, S. Karagadde, D. Rowley, T.J. Marrow, T. Connolley, P.D. Lee, Time-resolved synchrotron tomographic quantification of deformation-induced flow in a semi-solid equiaxed dendritic Al - Cu alloy, Scr. Mater. 103 (2015) 69-72. https://doi.org/10.1016/j.scriptamat.2015.03.011.

[32] D. Tourret, A. Karma, Growth competition of columnar dendritic grains: A phase-field study, Acta Mater. 82 (2015) 64-83. https://doi.org/10.1016/j.actamat.2014.08.049.

[33] H.B. Dong, P.D. Lee, Simulation of the columnar-to-equiaxed transition in directionally solidified Al-Cu alloys, Acta Mater. 53 (2005) 659-668. https://doi.org/10.1016/j.actamat.2004.10.019.

[34] L. Yuan, P.D. Lee, A new mechanism for freckle initiation based on microstructural level simulation, Acta Mater. 60 (2012) 4917-4926. https://doi.org/10.1016/j.actamat.2012.04.043. 
[35] T. Nelson, B. Cai, N. Warnken, P.D. Lee, E. Boller, O. V. Magdysyuk, N.R. Green, Gravity effect on thermal-solutal convection during solidification revealed by fourdimensional synchrotron imaging with compositional mapping, Scr. Mater. 180 (2020) 29-33. https://doi.org/10.1016/j.scriptamat.2019.12.026.

[36] B. Cai, A. Kao, P.D. Lee, E. Boller, H. Basevi, A.B. Phillion, A. Leonardis, K. Pericieous, Growth of $\beta$ intermetallic in an Al-Cu-Si alloy during directional solidification via machine learned 4D quantification, Scr. Mater. 165 (2019) 29-33.

https://doi.org/10.1016/j.scriptamat.2019.02.007.

[37] M. Drakopoulos, T. Connolley, C. Reinhard, R. Atwood, O. Magdysyuk, N. Vo, M. Hart, L. Connor, B. Humphreys, G. Howell, S. Davies, T. Hill, G. Wilkin, U. Pedersen, A. Foster, N. De Maio, M. Basham, F. Yuan, K. Wanelik, I12: the Joint Engineering, Environment and Processing (JEEP) beamline at Diamond Light Source, J. Synchrotron Radiat. 22 (2015) 1-10. https://doi.org/10.1107/S1600577515003513.

[38] N. Otsu, A threshold selection method from gray-level histograms, Syst. Man Cybern. IEEE Trans. 9 (1979) 62-66.

[39] W. Wang, P.D. Lee, M. McLean, A model of solidification microstructures in nickelbased superalloys: Predicting primary dendrite spacing selection, Acta Mater. 51 (2003) 2971-2987. https://doi.org/10.1016/S1359-6454(03)00110-1.

[40] A. Kao, B. Cai, P.D. Lee, K. Pericleous, The effects of Thermoelectric Magnetohydrodynamics in directional solidification under a transverse magnetic field, J. Cryst. Growth. 457 (2017) 270-274. https://doi.org/10.1016/j.jcrysgro.2016.07.003.

[41] A. Phillion, M. Založnik, I. Spindler, N. Pinter, C.-A. Aledo, H. Nguyen-Thi, G. Reinhart, G. Boussinot, M. Apel, H. Combeau, Evolution of a mushy zone in a static temperature gradient using an average volume approach, Acta Mater. in press (2017) 206-216. https://doi.org/10.1016/j.actamat.2017.09.011.

\section{Data availability}

Representative samples of the research data are given in the figures (and supplementary materials). Other datasets generated and/or analysed during this study are not publicly available due to their large size but are available from the corresponding authors on reasonable request.

\section{Acknowledgements}

This work was supported by the UK-EPSRC (EP/I02249X/1 and EP/K007734/1). We thank the beamtimes provided by the European Synchrotron Radiation Facility (MA2989) and Diamond Light Source (EE12631, EE13764 and EE19216-1). We appreciate staffs at the Diamond Light Source (Dr Thomas Connolley, Mr Bob Humphreys, and Mr Stuart Gurney) for support. The provision of the electromagnet by 116 beamline at the Diamond Light Source is acknowledged. B.C. thanks the support from the Diamond-Birmingham Collaboration and the Alan Turing Fellowship. PDL thanks the Royal Academy of Engineering for support (CiET1819/10). We greatly appreciate the assistance of beamtime experiments by many colleagues especially $\mathrm{Mr}$ Martin Kinsley (Materials Research Facility, UKAEA), Dr Andre Phillion (McMaster University, Canada), Dr Matthaios Alexandrakis (University of Greenwich, UK), Mr Ivars Krastins 
(University of Greenwich, UK), Mr Teddy Gan (University of Greenwich, UK) and Dr Enyu Guo (Dalian University of Technology, China). 

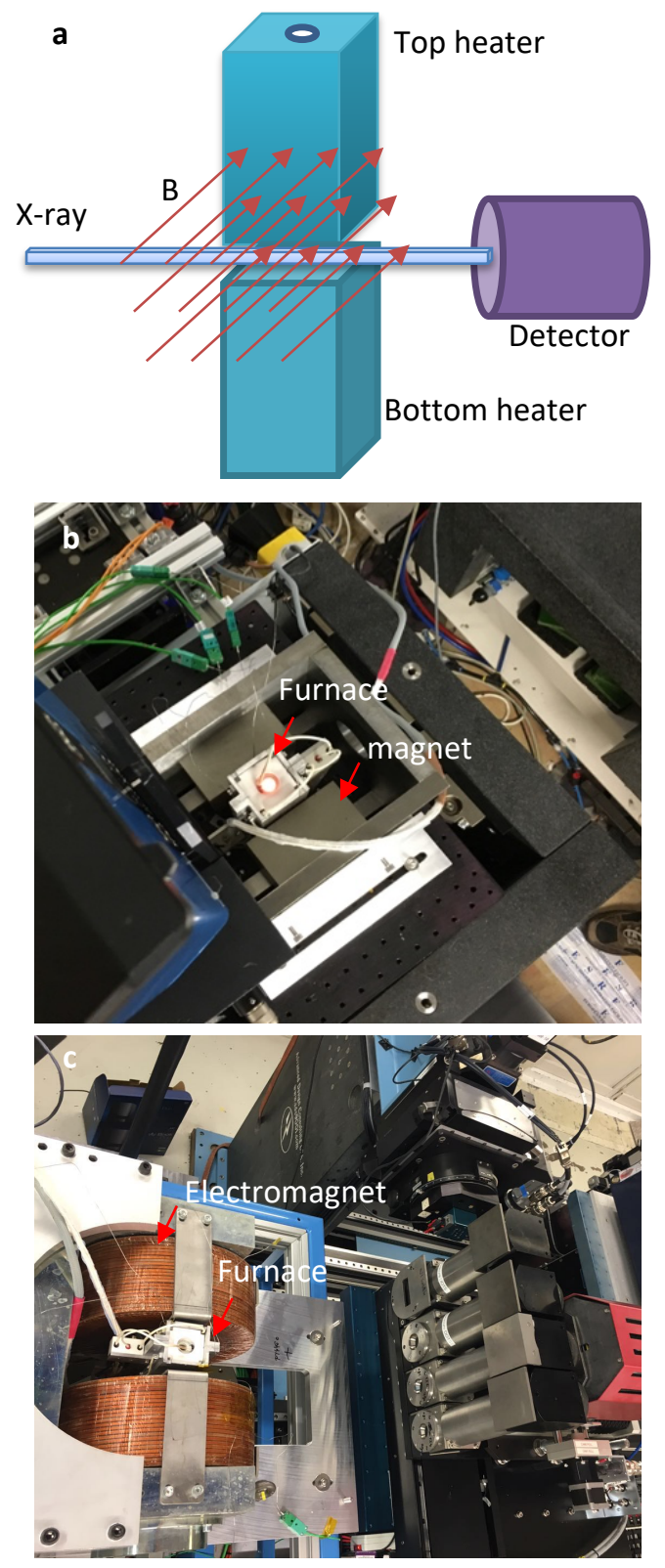

Fig. 1. (a) Schematic of the MagDS rig and experimental set-up. (b) The MagDS coupled with a permanent magnet york at ID19, European Synchrotron Radiation Facility (top view); (c) the MagDS with an electromagnet at I12, Diamond Light Source (top view). 


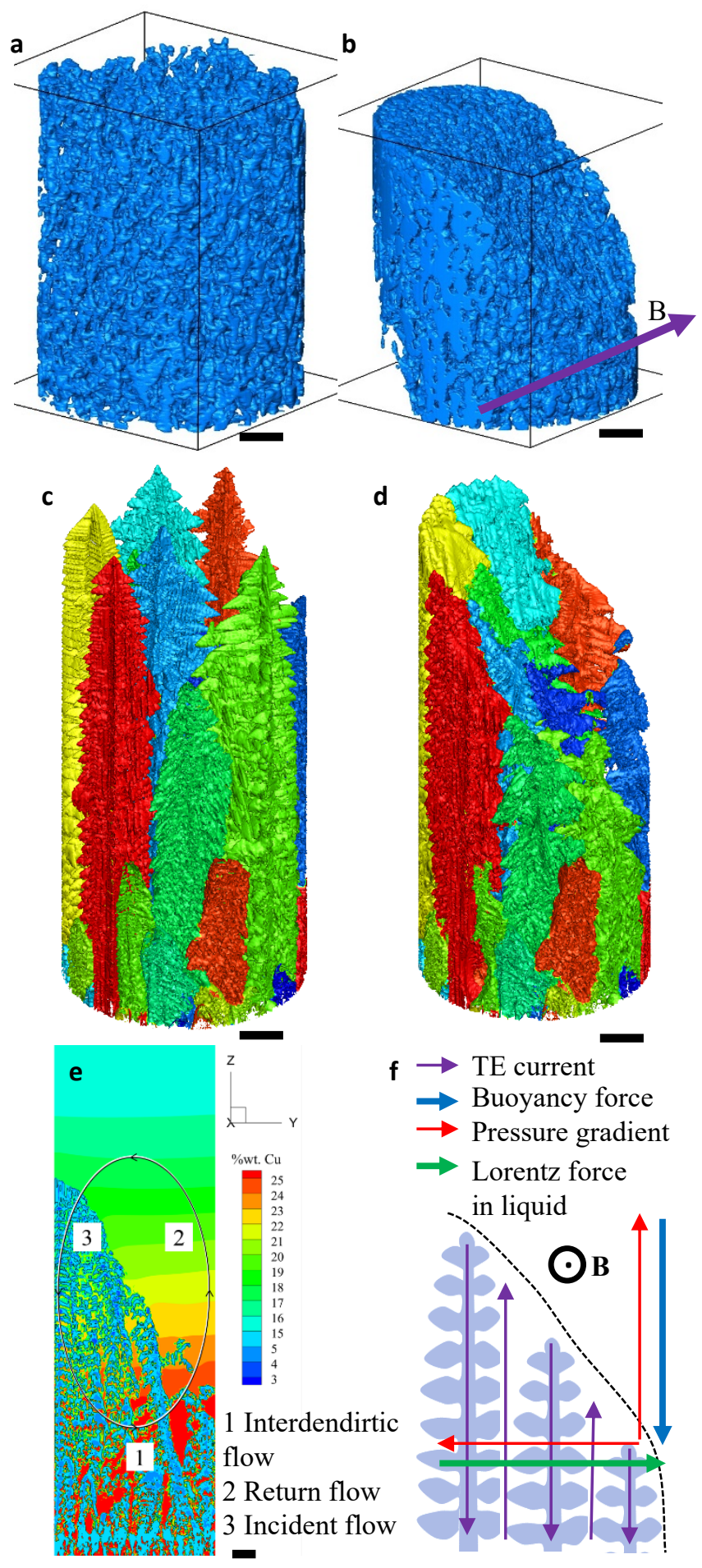

Fig.2. (a) The primary phase of directionally solidifying Al-15Cu sample held at a temperature gradient of $\mathbf{1 0}$ $\mathbf{K} / \mathbf{m m}$ for $\mathbf{1 0} \min (\mathrm{a}) \mathrm{B}=0 \mathrm{~T}$, then held at magnetic field of (b) $\mathbf{B}=0.5 \mathrm{~T}$ for $10 \mathrm{~min}$. Simulation results $(\mathrm{c}) \mathbf{B}=0 \mathrm{~T}$, (d) $\mathbf{B}=0.5 \mathrm{~T}$, (e) Solute concentration with $\mathbf{B}=0.5 \mathrm{~T}$ and (f) schematic of mechanism with $\mathbf{B}=0.5 \mathrm{~T}$. All scalebars are $300 \mu \mathrm{m}$ 

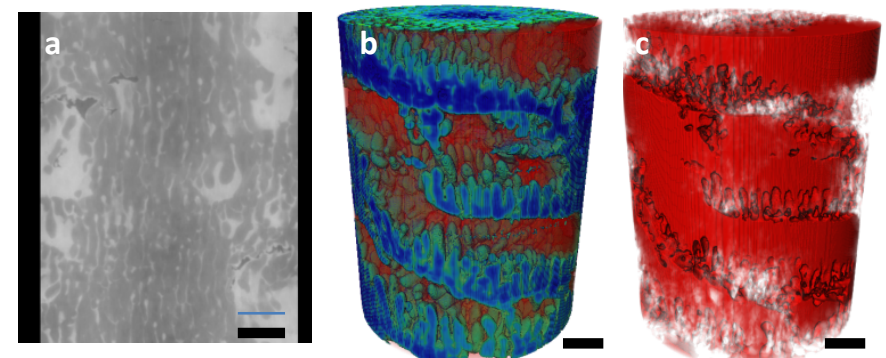

$\Delta \mathrm{T}=10 \mathrm{~K} / \mathrm{mm} ; \mathrm{CR}=0.02 \mathrm{~K} / \mathrm{s} ; \mathrm{B}=0.5 \mathrm{~T}$
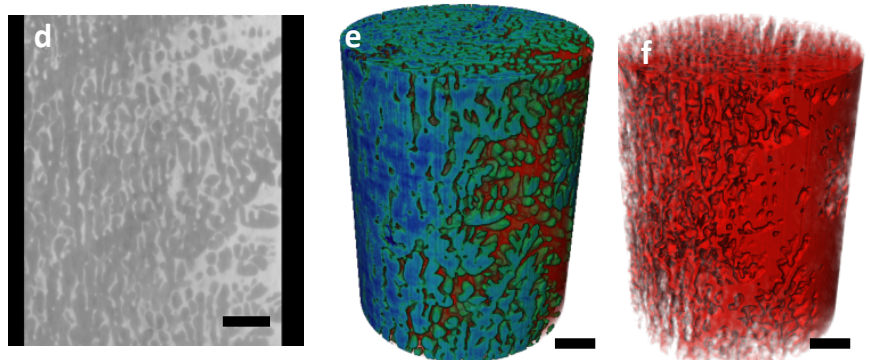

$\Delta \mathrm{T}=10 \mathrm{~K} / \mathrm{mm} ; \mathrm{CR}=0.05 \mathrm{~K} / \mathrm{s} ; \mathrm{B}=0 \mathrm{~T}$

Fig.3 Microstructures with and without the application of magnetic field. (a) to (c) directional solidified Al-15Cu alloy rotating within a $0.5 \mathrm{~T}$ transversal magnetic field under $\sim 10$ $\mathrm{K} / \mathrm{mm}$ temperature gradient $(\Delta \mathrm{T})$, and $\sim 0.02 \mathrm{~K} / \mathrm{s}$ cooling rate $(C R):(a)$ the vertical slice extracted from the $3 \mathrm{D}$ tomography; $3 \mathrm{D}$ volume rendering of final microstructures showing the screw solid phase region (the blue and green color in (b)) and the channel of eutectic phase (red color in (b) and (c)). (d) to (f) directional solidified Al-15Cu alloy without magnetic field $(10 \mathrm{~K} / \mathrm{mm}$ temperature gradient, $0.02 \mathrm{~K} / \mathrm{s}$ cooling rate and $0 \mathrm{~T}$ magnetic field). (Scale bar: $300 \mu \mathrm{m}$ ). 
a

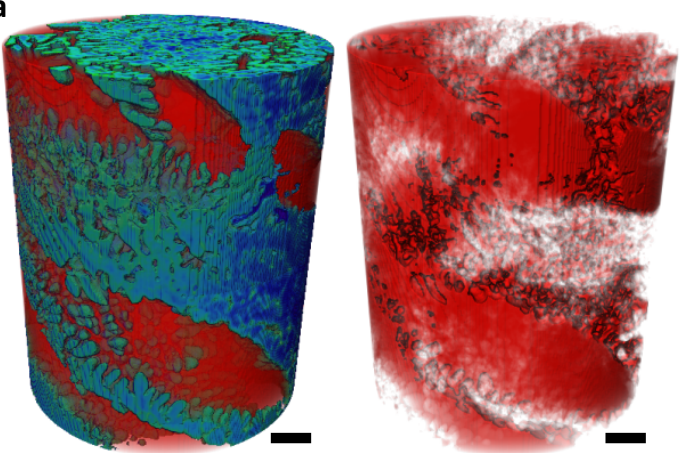

$\mathrm{G}=5 \mathrm{~K} / \mathrm{mm} ; \mathrm{CR}=0.05 \mathrm{~K} / \mathrm{s} ; \mathrm{B}=0.5 \mathrm{~T}$

b
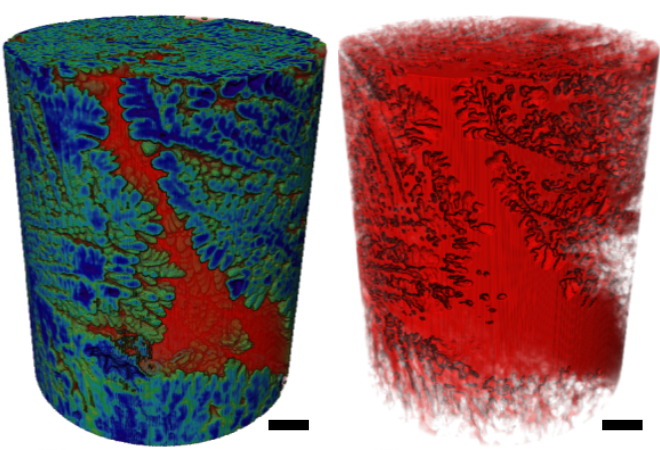

$\mathrm{G}=10 \mathrm{~K} / \mathrm{mm} ; \mathrm{CR}=\sim 0.25 \mathrm{~K} / \mathrm{s} ; \mathrm{B}=0.5 \mathrm{~T}$

Fig. 4. The formation of screw structure under different thermal conditions at magnetic field of $0.5 \mathrm{~T}$ : (a) $\mathrm{G}=5 \mathrm{~K} / \mathrm{mm}, \mathrm{CR}=0.05 \mathrm{~K} / \mathrm{s}, \mathrm{B}=0.5$ $\mathrm{T}$; (b) $\mathrm{G}=10 \mathrm{~K} / \mathrm{mm}, \mathrm{CR}=\sim 0.25 \mathrm{~K} / \mathrm{s}, \mathrm{B}=0.5 \mathrm{~T}$. (scale bar $300 \mu \mathrm{m}$ ) 

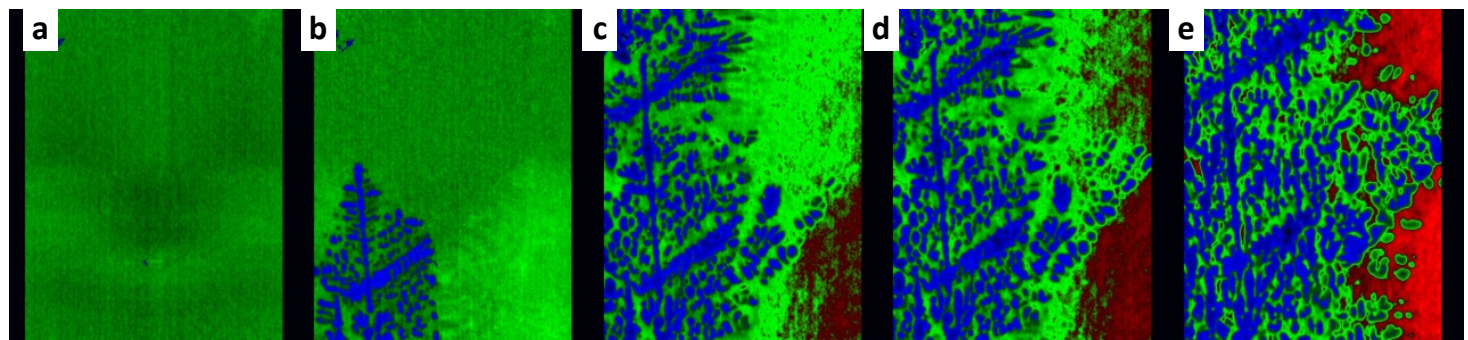

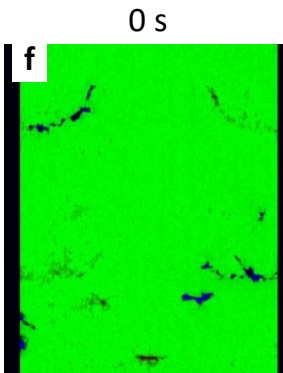

$0 \mathrm{~s}$

$560 \mathrm{~s}$

$640 \mathrm{~s}$

$1120 \mathrm{~s}$
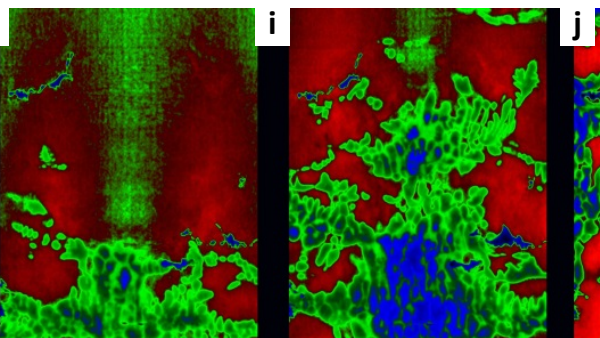

$1120 \mathrm{~s}$

$1920 \mathrm{~s}$

5.5

$33(w t \% C u)$

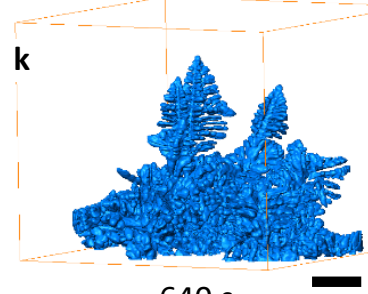

$640 \mathrm{~s}$

n

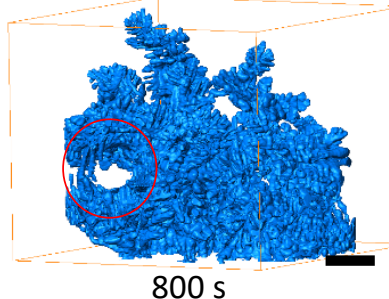

$800 \mathrm{~s}$

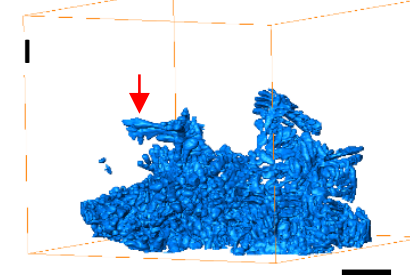

$688 \mathrm{~s}$

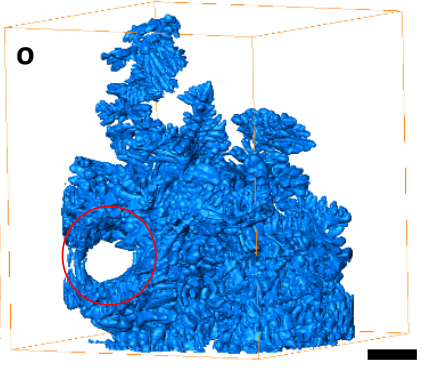

$880 \mathrm{~s}$

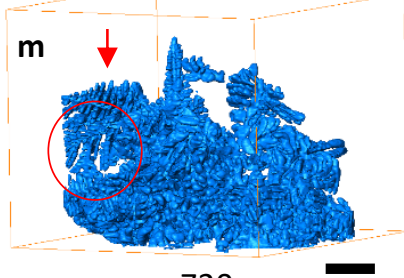

$720 \mathrm{~s}$

p

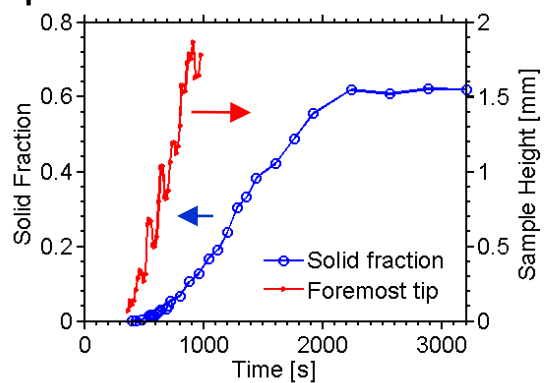

Fig. 5. In situ view of compositional and structural evolution during solidification of Al-15Cu sample: (a to j) vertical slices color-coded by Cu concentration: (a to e) $\Delta T=10 \mathrm{~K} / \mathrm{mm} ; \mathrm{CR}=0.05 \mathrm{~K} / \mathrm{s} ; \mathrm{B}=0 \mathrm{~T}$; (f to j) $\Delta T=10 \mathrm{~K} / \mathrm{mm} ; C R=0.02 \mathrm{~K} / \mathrm{s} ; \mathrm{B}=0.5 \mathrm{~T}$. (k-o) in situ view of the solid phase growth at different time; and (p) the evolution of the solid fraction and the height of the foremost dendrite tip as a function of time. (scale bar: $300 \mu \mathrm{m}$ ) 


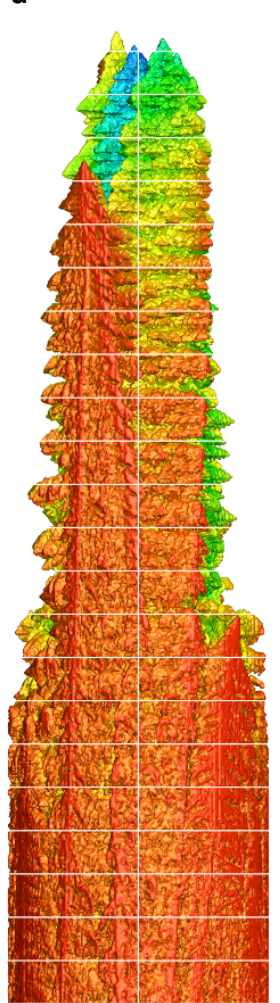

TE

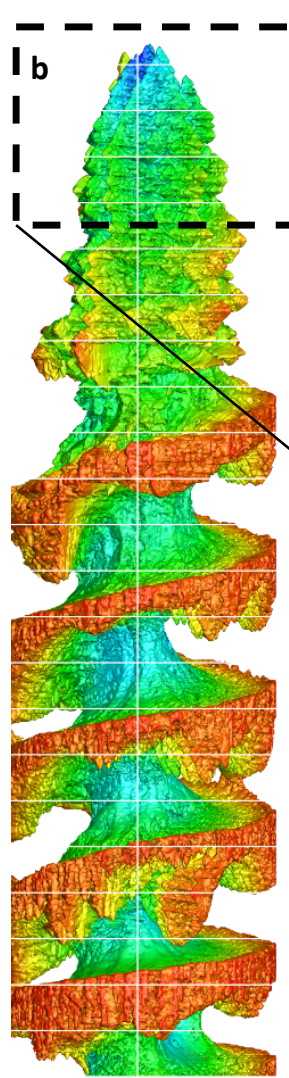

EMS

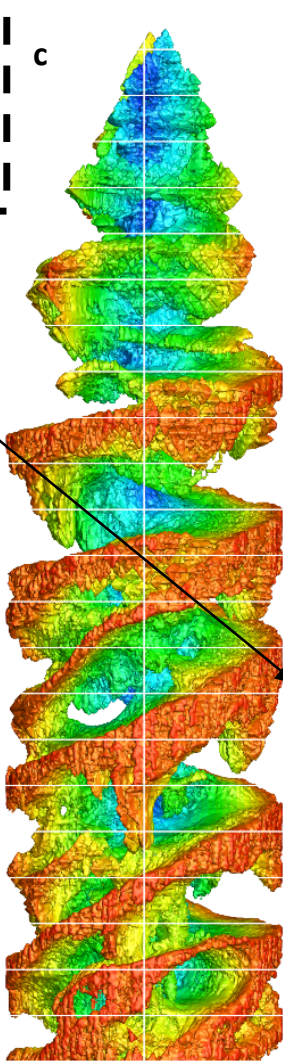

EMS+TE

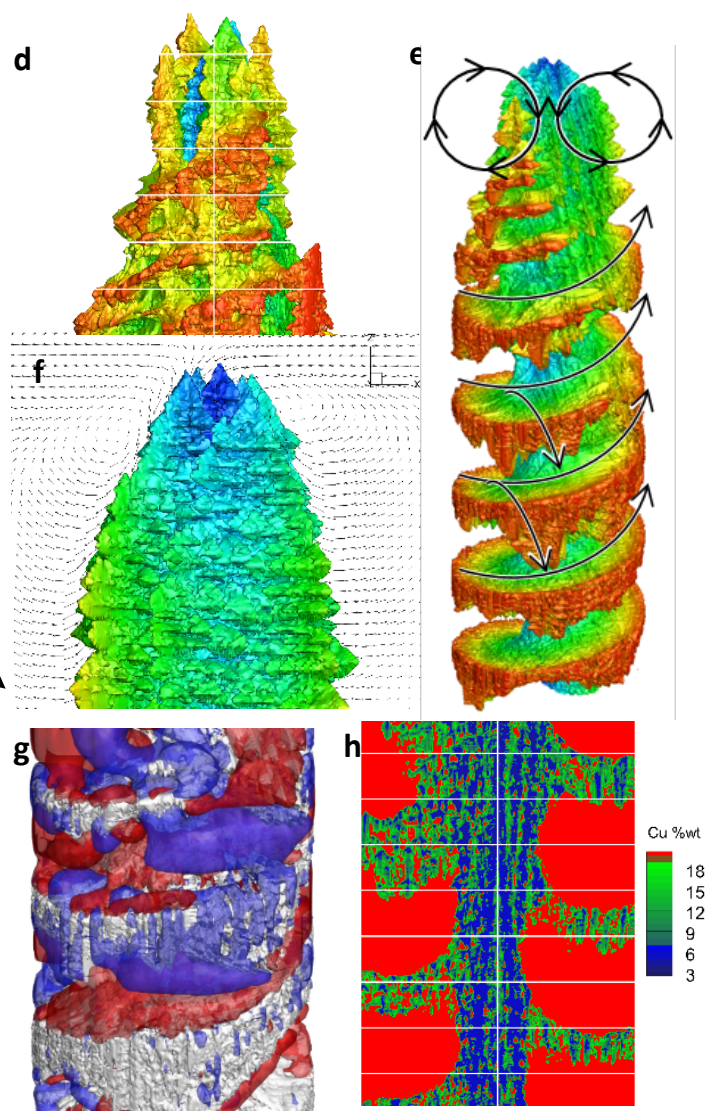

Fig. 6. Simulation results of sample rotation in a transversal magnetic field. Comparison of TEMHD and EMS effect (a) structure with only TEMHD, i.e. EMD from rotation is neglected; (b) structure with only EMS i.e. Seebeck coefficient $S=0$, (c) structure with both TE and EMS. (d) Interface structure showing initiation of screw when B turns on for $200 \mathrm{~s}$. (f) flow field at time $1000 \mathrm{~s}$ at the tip of the screw. (g) Formation of screw thread at time $1000 \mathrm{~s}$. (e) schematic showing the liquid flow path. (g) Iso-surfaces of liquid flow at time $1000 \mathrm{~s}(+10 \mu \mathrm{m} / \mathrm{s}$ (red) and $-10 \mu \mathrm{m} / \mathrm{s}$ (blue) flow velocity parallel to the thermal gradient); (h) solute composition map at time $1000 \mathrm{~s}$. 
a
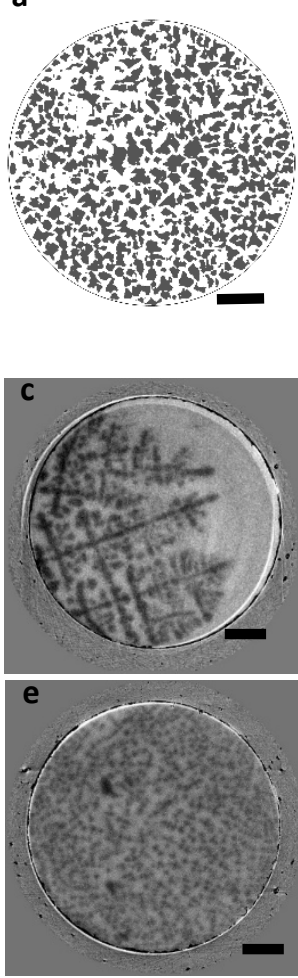

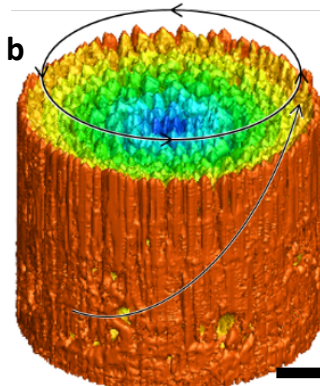

d

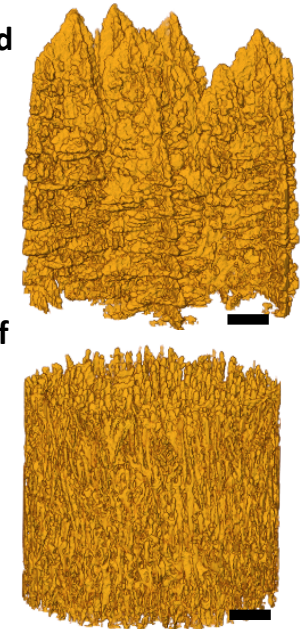

Fig. 7. Solidifying sample with a higher Seebeck power rotating within transverse magnetic field. (a) and (b) Simulated results of rotating sample solidifying in $\mathbf{B}=0.5 \mathrm{~T}$ with an increased Seebeck power $\left(\mathrm{S}=10^{-5} \mathrm{~V} / \mathrm{K}\right)$; (c) to ( $\left.\mathrm{f}\right)$,

Directionally solidifying Al-3.5Cu-5.5Si alloy; (c) and (d) without magnetic field; (e) and (f) with magnetic field of $\mathbf{B}=0.5 \mathrm{~T}$. (a, $\mathrm{c}$ and e) horizontal slice, (b, $d$ and f) 3D volume rendering. (Scale bar $300 \mu \mathrm{m}$ ) 